\title{
Abundant Coexisting Multiple Attractors' Behaviors in Three-Dimensional Sine Chaotic System
}

\author{
Huagan Wu (D), ${ }^{1}$ Han Bao $\mathbb{D D}^{2}$ Quan Xu ${ }^{2}{ }^{1}$ and Mo Chen ${ }^{1}{ }^{1}$ \\ ${ }^{1}$ School of Information Science and Engineering, Changzhou University, Changzhou 213164, China \\ ${ }^{2}$ College of Automation Engineering, Nanjing University of Aeronautics and Astronautics, Nanjing 211106, China
}

Correspondence should be addressed to Mo Chen; mchen@cczu.edu.cn

Received 15 October 2019; Revised 2 November 2019; Accepted 15 November 2019; Published 6 December 2019

Guest Editor: Viet-Thanh Pham

Copyright ( $\odot 2019$ Huagan Wu et al. This is an open access article distributed under the Creative Commons Attribution License, which permits unrestricted use, distribution, and reproduction in any medium, provided the original work is properly cited.

This paper presents a novel and simple three-dimensional (3-D) chaotic system by introducing two sine nonlinearities into a simple 3-D linear dynamical system. The presented sine system possesses nine equilibrium points consisting of five index-2 saddle foci and four index-1 saddle foci which allow the coexistence of various types of disconnected attractors, also known as multistability. The coexisting multiple attractors are depicted by the phase plots and attraction basins. Coexisting bifurcation modes triggered by different initial values are numerically simulated by two-dimensional bifurcation and complexity plots under two sets of initial values and one-dimensional bifurcation plots under three sets of initial values, which demonstrate that the abundant coexisting multiple attractors' behaviors in the presented sine system are related not only to the system parameters but also to the initial values. A simulation-oriented circuit model is synthesized, and PSIM (power simulation) screen captures well validate the numerical simulations.

\section{Introduction}

Recently, numerous nonlinear dynamical systems have been reported that they present the coexistence of two or more disconnected attractors with their isolated attraction basins. The coexisting phenomena of two or more attractors have been encountered in nonlinear oscillating circuits [1-5], biological neuron models $[6,7]$, Hopfield neural networks [8-11], vibroimpact system [12], superconducting quantum interference device oscillators [13], pure mathematical systems [14-17], and so on. This striking phenomenon, also known as multistability, demonstrates that the system initial values do play an important role in the emergence of complex coexisting attractors' behaviors [18, 19]. For a multistable dynamical system, it is usually challenging to predict the final steady state to which the dynamical system will tend for a given initial value since a small disturbance in the initial value can alter the steady state of such dynamical systems [20-23]. Multistability has great application potentials in the chaos-based secure communication and information encryption [24-27], but efficient prediction and control methods should be employed to make these dynamical systems in the desired oscillating modes [28-32].

Usually, an effective method for implementing the initialrelated multistability is to lead one, two, or more generic or extended memristors in various existing circuits and systems [15-17, 33-36]. Memristor-based circuits and systems with different types of equilibrium points are easy to exhibit coexisting attractors' behaviors of multistability. Comparatively speaking, another beneficial and simple method for generating initial value offset-boosted coexisting attractors is to put periodic trigonometric functions into specific offsetboostable dynamical systems [37-41]. When the cyclic periods for the periodic functions are identical, any attractor will be copied by periodic offset boosting the initial values [37]. However, due to the reported offset-boostable dynamical systems with self-contained nonlinearities, the newly constructing multistable dynamical systems become relatively complicated [38-41], not convenient for theoretical analyses and hardware circuit implementations. The algebraic simplicity of system's structure and topological complexity of chaotic attractors are benefits for developing chaos-based 
cryptosystems [42]. In this paper, based on a simple 3-D linear dynamical system and two newly introduced sine nonlinearities, a novel and extremely simple $3-\mathrm{D}$ sine chaotic system is readily constructed, from which abundant coexisting multiple attractors' behaviors are observed [43].

The rest is organized as follows. In Section 2, a novel and simple 3-D sine chaotic system is presented. It has nine equilibrium points consisting of five index-2 saddle foci and four index-1 saddle foci, resulting in the coexistence of up to six types of disconnected attractors. In Section 3, by twodimensional bifurcation and complexity plots under two sets of initial values and one-dimensional bifurcation plots under three sets of initial values, coexisting bifurcation modes are numerically simulated to demonstrate the abundant coexisting multiple attractors' behaviors. In Section 4, with the simulation-oriented circuit model, PSIM screen captures validate the numerical simulations. The conclusion is summarized in Section 5.

\section{System Model and Its Coexisting Multiple Attractors}

By introducing two sine nonlinearities with two coupling coefficients into a simple 3-D linear dynamical system, a novel 3-D sine chaotic system with simple algebraic equations is easily achieved, which is modeled by

$$
\begin{aligned}
& \dot{x}=y+z-k_{1} \sin (y), \\
& \dot{y}=-x+z, \\
& \dot{z}=-x-z+k_{2} \sin (x),
\end{aligned}
$$

where $x, y$, and $z$ are the three state variables and $k_{1}$ and $k_{2}$ are the two positive constants.

The presented sine system in (1) is symmetric about the origin and dissipative. The symmetric property can be demonstrated by the invariance of system (1) with respect to the transformation $(x, y, z) \longleftrightarrow(-x,-y,-z)$. The dissipativity is explained by

$$
\nabla V=\frac{\partial \dot{x}}{\partial x}+\frac{\partial \dot{y}}{\partial y}+\frac{\partial \dot{z}}{\partial z}=-1<0 .
$$

Thus, the orbits are finally confined to a specific subset with zero volume and its asymptotic motion settles onto a standalone attractor.

The equilibrium points of the presented sine system in (1) are obtained by solving the following equations:

$$
\begin{aligned}
& 0=y+z-k_{1} \sin (y), \\
& 0=-x+z, \\
& 0=-x-z+k_{2} \sin (x),
\end{aligned}
$$

which is expressed as

$$
E=(\delta, \sigma, \delta) .
$$

The values $\delta$ and $\sigma$ can be yielded by solving the following transcendental functions:

$$
h_{1}=2 \delta-k_{2} \sin (\delta)=0
$$

$$
h_{2}=\sigma+\delta-k_{1} \sin (\sigma)=0 \text {, }
$$

respectively.

The Jacobian matrix $\mathbf{J}$ at $E$ is given as

$$
\mathbf{J}=\left[\begin{array}{ccc}
0 & 1-k_{1} \cos (\sigma) & 1 \\
-1 & 0 & 1 \\
-1+k_{2} \cos (\delta) & 0 & -1
\end{array}\right],
$$

so that the characteristic polynomial is derived as

$$
P(\lambda)=\lambda^{3}+c_{1} \lambda^{2}+c_{2} \lambda+c_{3}=0,
$$

where

$$
\begin{aligned}
& c_{1}=1, \\
& c_{2}=2-k_{1} \cos (\sigma)-k_{2} \cos (\delta), \\
& c_{3}=\left[1-k_{1} \cos (\sigma)\right]\left[2-k_{2} \cos (\delta)\right] .
\end{aligned}
$$

The above characteristic polynomial implies that Jacobian matrix (7) has three nonzero roots. For these roots, Routh-Hurwitz conditions are given as

$$
\begin{aligned}
c_{1} & >0, \\
c_{3} & >0, \\
c_{1} c_{2}-c_{3} & >0,
\end{aligned}
$$

i.e.,

$$
\begin{aligned}
{\left[1-k_{1} \cos (\sigma)\right]\left[2-k_{2} \cos (\delta)\right] } & >0, \\
k_{1} \cos (\sigma)\left[1-k_{2} \cos (\delta)\right] & >0 .
\end{aligned}
$$

If the conditions in (11) are satisfied, i.e., $k_{1} \cos (\sigma)<1$ and $k_{2} \cos (\delta)<1, E$ is stable, leading to the existence of the point attractor. Otherwise, if any one of the conditions in (11) is not satisfied, i.e., $k_{1} \cos (\sigma)<1$ or $k_{2} \cos (\delta)<1, E$ is unstable, resulting in that unstable behaviors may be triggered in the presented sine system.

Denote $k_{1}=k_{2}=k$ and take $k=3.6$ and 5 as two examples. The values $\delta$ and $\sigma$ of the equilibrium point $E$ in (4) are the intersection points of two function curves $h_{1}$ and $h_{2}$ described by (5) and (6), as shown in Figures 1(a) and 1(b), respectively, from which nine pairs of $\delta$ and $\sigma$ are obtained by inspecting the intersection points, indicating the existence of nine equilibrium points in the presented sine system. With these equilibrium points, the three nonzero eigenvalues are calculated from the Jacobian matrix $\mathbf{J}$ in (7) and the corresponding stabilities can be determined, as listed in Table 1. The calculation results illustrate that five index-2 saddle foci (Index-2 USF, for short) and four index-1 saddle foci (Index-1 USF, for short) can be found, which could emerge five disconnected attracting regions when these attracting regions cannot be linked with each other, leading to the coexistence of disconnected attractors.

For $k=3.6,5$, and several sets of the initial values (labeled in Figure 2), the phase plots of coexisting multiple attractors projected on the $x-y$ plane are depicted in Figures 2(a) and 2(b), respectively. In Figure 2(a), a chaotic attractor coexisted with twin small-size period-1 limit cycles, twin large-size 


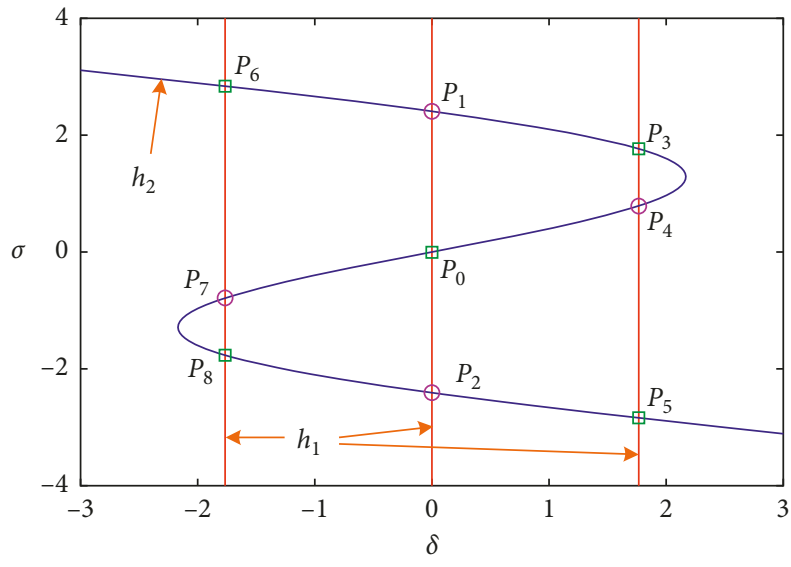

口 Index-2 USF

O Index-1 USF

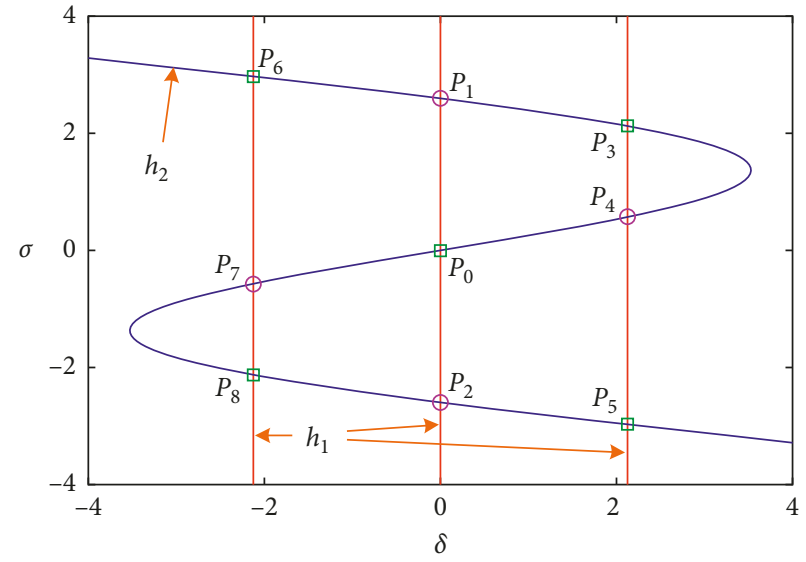

口 Index-2 USF

O Index-1 USF

(a)

(b)

Figure 1: Values $\delta$ and $\sigma$ of the equilibrium points by inspecting the intersections of two function curves $h_{1}$ and $h_{2}$ described by (5) and (6). (a) $k=3.6$. (b) $k=5$.

TABLE 1: Equilibrium points, eigenvalues, and stabilities for $k=3.6$ and 5 .

\begin{tabular}{cccc}
\hline$k$ & Equilibrium points & Eigenvalues & Stabilities \\
\hline \multirow{4}{*}{3.6} & $P_{0}:(0,0,0)$ & $1.0528 \pm j 0.4807,-3.1057$ & Index-2 USF \\
& $P_{1,2}:(0, \pm 2.4074,0)$ & $1.3656,-1.1828 \pm j 1.7041$ & Index-1 USF \\
& $P_{3,8}:( \pm 1.7659, \pm 1.7668, \pm 1.7659)$ & $0.1205 \pm j 1.9193,-1.2410$ & Index-2 USF \\
& $P_{4,7}:( \pm 1.7659, \pm 0.7858, \pm 1.7659)$ & $1.3103,-1.1551 \pm j 1.3587$ & Index-1 USF \\
& $P_{5,6}:( \pm 1.7659, \pm 2.8378, \pm 1.7659)$ & $0.3285 \pm j 2.6672,-1.6569$ & Index-2 USF \\
\hline & $P_{0}:(0,0,0)$ & $1.4346 \pm j 1.0215,-3.8692$ & Index-2 USF \\
5 & $P_{1,2}:(0, \pm 2.5976,0)$ & $2.0719,-1.5359 \pm j 2.2986$ & Index-1 USF \\
& $P_{3,8}:( \pm 2.1253, \pm 2.1271, \pm 2.1253)$ & $0.4429 \pm j 2.9575,-1.8857$ & Index-2 USF \\
& $P_{4,7}:( \pm 2.1253, \pm 0.5756, \pm 2.1253)$ & $2.1115,-1.5558 \pm j 2.1419$ & Index-1 USF \\
& $P_{5,6}:( \pm 2.1253, \pm 2.9680, \pm 2.1253)$ & $0.6154 \pm j 3.4532,-2.2309$ & Index-2 USF \\
\hline
\end{tabular}

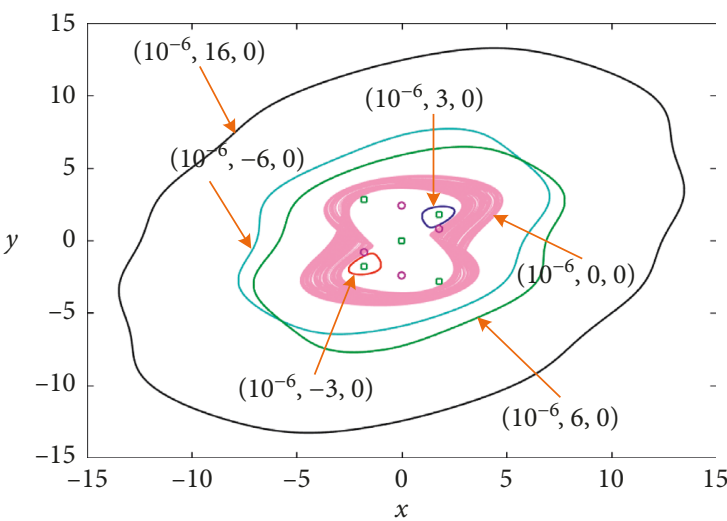

(a)

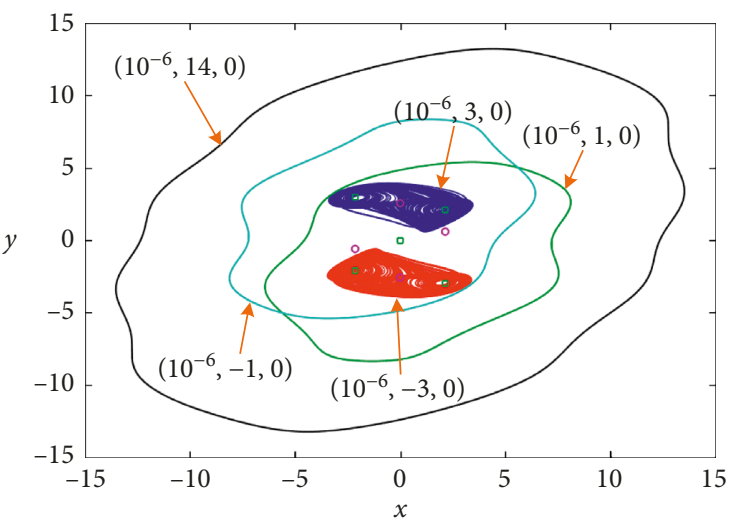

(b)

FIgURe 2: Phase plots of coexisting multiple attractors projected on the $x-y$ plane for different initial values. (a) For $k=3.6$, six types of coexisting multiple attractors. (b) For $k=5$, five types of coexisting multiple attractors.

period-1 limit cycles, and a relatively larger size period-1 limit cycle. By contrast, in Figure 2(b), two chaotic attractors coexisted with twin large-size period-1 limit cycles and a relatively larger size period-1 limit cycle. Therefore, up to six types of coexisting multiple attractors are numerically disclosed in the 3-D sine chaotic system because of the attracting and repelling interactions between the five index- 2 saddle foci and four index-1 saddle foci. 
To detect the attracting regions of the coexisting multiple attractors given in Figure 2, the attraction basins in the initial value plane are used to classify different dynamical behaviors [44]. For the two coupling coefficients used in Figure 2, the attraction basins in the $x(0)-y(0)$ plane with $z(0)=10^{-6}$ are depicted in Figure 3. The attracting regions painted by different colors represent the initial value regions corresponding to different long-term oscillating states, i.e., coexisting multistable states, which are identical with the colored trajectories appearing in Figure 2. Thus, the attraction basins show the relatively complicated manifold structures along with basin boundaries. Meanwhile, the numerical results in Figure 3 demonstrate the emergence of multistability in the presented sine system.

It can be concluded that due to the appearance of five index- 2 saddle foci and four index- 1 saddle foci and their interactions with each other, some disconnected attracting regions are thereby formed in the neighborhoods around these unstable saddle foci, resulting in the generation of coexisting multiple disconnected attractors.

\section{Initial Values-Related Coexisting Multiple Bifurcation Modes}

Because the presented sine system in (1) is symmetric about the origin, the disconnected attracting regions have locally symmetric behaviors, which are well exhibited in Figures 2 and 3 . For this reason and convenient analysis, three sets of the initial values $\left(10^{-6}, 0,0\right),\left(10^{-6}, 3,0\right)$, and $\left(10^{-6},-3,0\right)$ are considered in next numerical plots. The fourth-order Runge-Kutta algorithm with the $0.01 \mathrm{~s}$ time step and (700 s, $800 \mathrm{~s}$ ) time interval is used for depicting the phase plots and bifurcation plots, whereas the fourth-order Runge-Kuttabased Wolf's method with the $0.01 \mathrm{~s}$ time step and $20 \mathrm{ks}$ time end is adopted for calculating the Lyapunov exponents.

Firstly, two-dimensional bifurcation plots (bifurcation diagrams and dynamical maps) [23] are employed to show complex dynamical behaviors in the presented sine system intuitively, as shown in Figures 4 and 5. Here, both the coupling coefficients $k_{1}$ and $k_{2}$ are simultaneously increased in the region $[2,8]$ and two sets of initial values $\left(10^{-6}, 0,0\right)$ and $\left(10^{-6}, 3,0\right)$ are chosen. Note that the exhibited twodimensional bifurcation behaviors are similar to each other for the initial values $\left(10^{-6}, 3,0\right)$ and $\left(10^{-6},-3,0\right)$ due to the system symmetry.

As shown in Figure 4, the two-dimensional bifurcation diagrams in the $k_{1}-k_{2}$ parameter plane are obtained by calculating the periodicities of state variable $x$, which demonstrate rich and complex coexisting dynamical behaviors related to system parameters and initial values. The stable points and chaotic attractors distribute in the blackand red-colored regions, respectively, and the periodic attractors with different periodicities situate in the other colored regions. Comparing Figure 4(b) with Figure 4(a), there is a big difference between the two dynamical behaviors in the lower right regions, which is triggered by the initial values-dependent multistability in the presented sine system, leading to the coexistence of multiple bifurcation modes.
As shown in Figure 5, the two-dimensional dynamical maps in the $k_{1}-k_{2}$ parameter plane under two sets of initial values are depicted by evaluating the values of the largest Lyapunov exponent. The yellow-red-white colored regions with different positive values of the largest Lyapunov exponent represent different chaotic behaviors, the blackcolored regions with different negative values of the largest Lyapunov exponent only stand for stable point behaviors, and the black-yellow colored regions with the zero largest Lyapunov exponent represent different periodic behaviors. In a similar manner, the dynamical behaviors described by the dynamical maps in Figures 5(a) and 5(b) are of great difference, which manifest how coexisting dynamical behaviors evolve for different initial values.

Similarly, the two-dimensional spectral entropy-based complexity plots in the $k_{1}-k_{2}$ parameter plane are displayed in Figure 6, where two sets of initial values $\left(10^{-6}, 0,0\right)$ and $\left(10^{-6}, 3,0\right)$ are employed. On the basis of the Fourier transform [32, 45], the complexity values are obtained by calculating the spectral entropy of the time sequence of the variable $x$. The relatively large complexity value in Figure 6 indicates the appearance of an irregularly chaotic sequence, whereas the relatively small complexity value in Figure 6 represents the occurrence of a regularly periodic sequence. For the two sets of different initial values, there are some differences in the complexity plots between Figures 6(a) and 6(b), implying that the system initial values have great effects on the dynamical behaviors of the presented sine system.

Therefore, the dynamical maps shown in Figure 5 and complexity plots shown in Figure 6 can reflect the dynamical evolutions with the variations of the system parameters and initial values, which are the effective supplements to confirm the coexisting dynamical behaviors depicted by the bifurcation diagrams in Figure 4.

To visualize the coexisting multiple bifurcation modes related to the initial values, three sets of initial values $\left(10^{-6}, 0\right.$, $0),\left(10^{-6}, 3,0\right)$, and $\left(10^{-6},-3,0\right)$ are considered and both the coupling coefficients $k_{1}$ and $k_{2}$ are simultaneously increased in the region $[2,8]$. Denote $k_{1}=k_{2}=k$ as a bifurcation parameter. The one-dimensional bifurcation plots with the variation of the system parameter $k$ are shown in Figure 7. In Figure 7(a), the bifurcation diagrams drawn by the black, blue, and red trajectories correspond to those initiated from the initial values $\left(10^{-6}, 0,0\right),\left(10^{-6}, 3,0\right)$, and $\left(10^{-6},-3,0\right)$, respectively. And in Figure 7(b), the first two Lyapunov exponents associated with three sets of initial values are drafted in the upper, middle, and bottom of Figure 7(b), which entirely match with the bifurcation diagrams in Figure 7(a). Therefore, when more initial values are considered, more complicated coexisting multiple bifurcation modes can be revealed in the presented sine system.

Observed from Figure 7, abundant coexisting multiple attractors' behaviors related to the initial values are exhibited, including stable points, periodic oscillations, and chaotic oscillations along with period-doubling bifurcations, tangent bifurcations, and crisis scenarios. When two sets of initial values $\left(10^{-6}, 3,0\right)$ and $\left(10^{-6},-3,0\right)$ are chosen, both the depicted dynamical behaviors in Figure 7 are basically identical over the entire parameter region, with only slight 


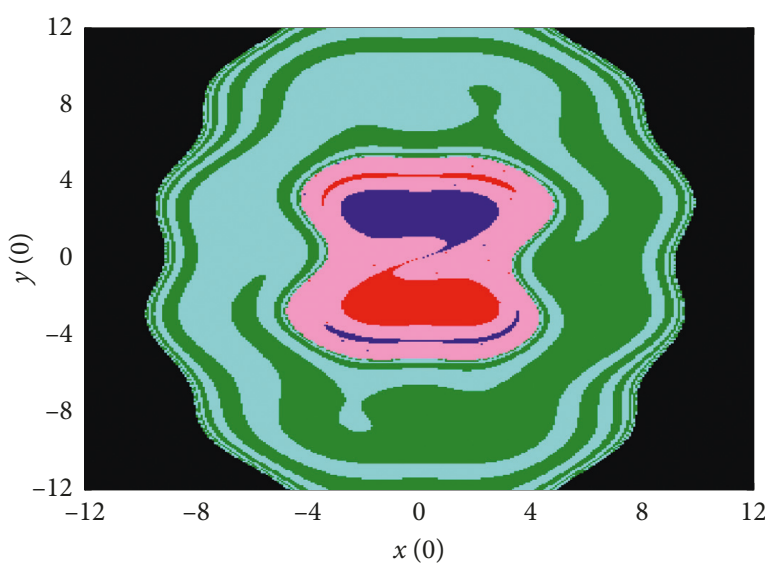

(a)

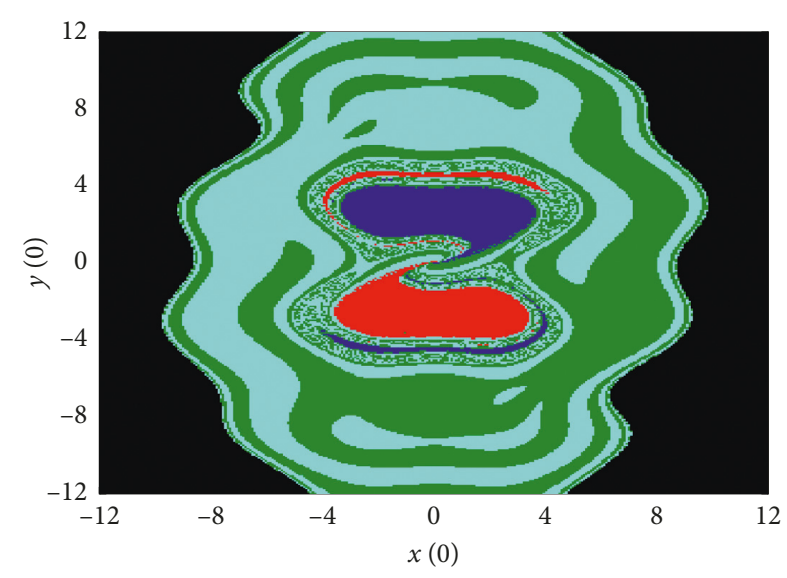

(b)

Figure 3: Two attraction basins in the $x(0)-y(0)$ plane with $z(0)=10^{-6}$ and the painted colors correspond to the colored motion orbits shown in Figure 2. (a) Attraction basin for $k=3.6$. (b) Attraction basin for $k=5$.

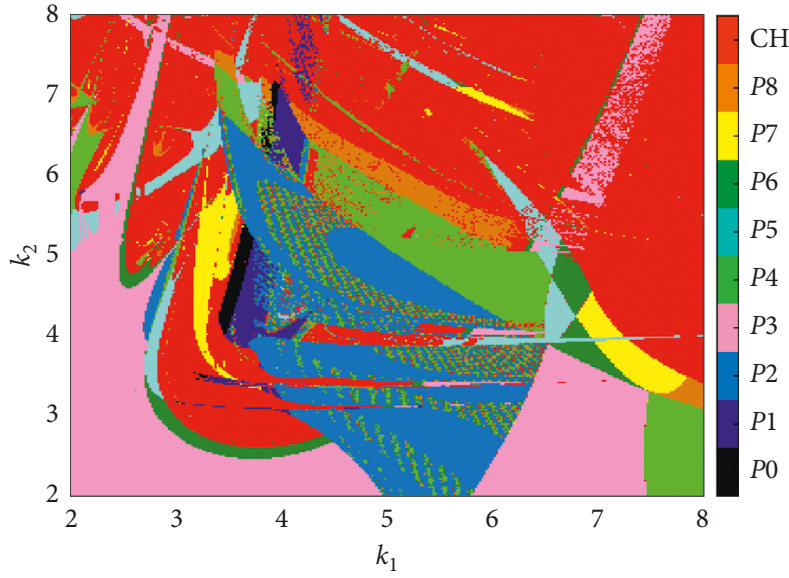

(a)

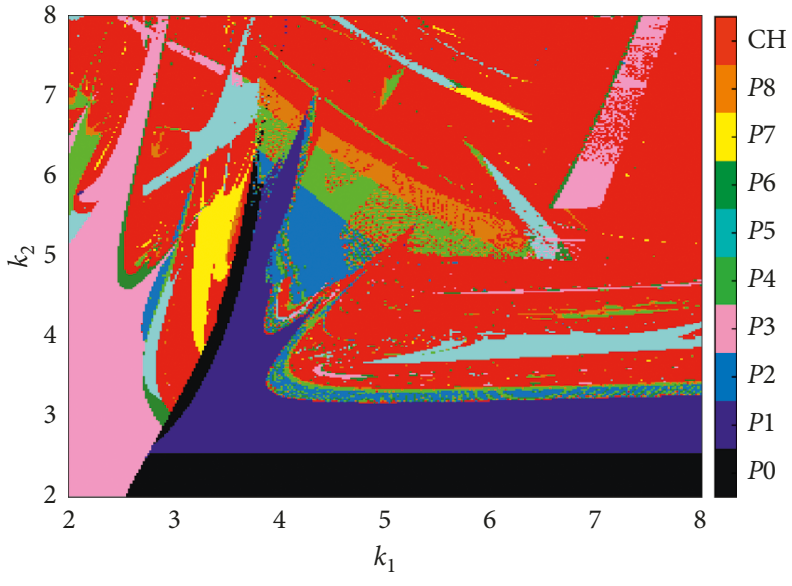

(b)

FIgURE 4: Two-dimensional bifurcation diagrams in the $k_{1}-k_{2}$ parameter plane through calculation of the periodicities of the state variable $x$ under two sets of initial values. (a) Initial values $\left(10^{-6}, 0,0\right)$. (b) Initial values $\left(10^{-6}, 3,0\right)$.

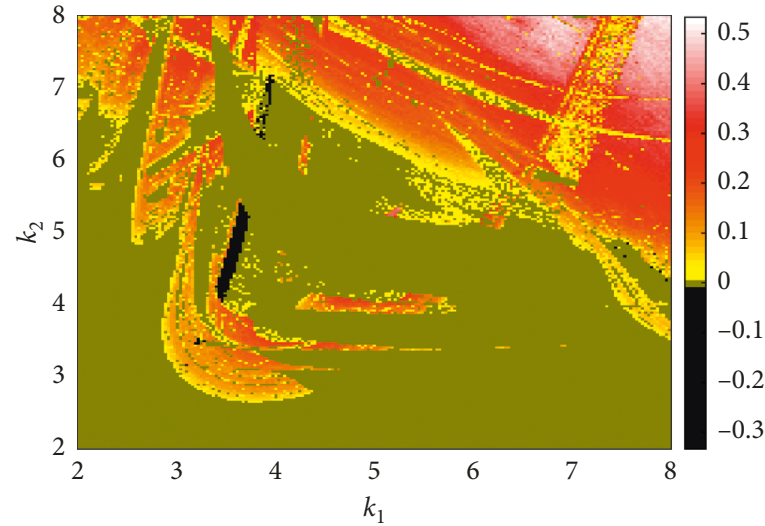

(a)

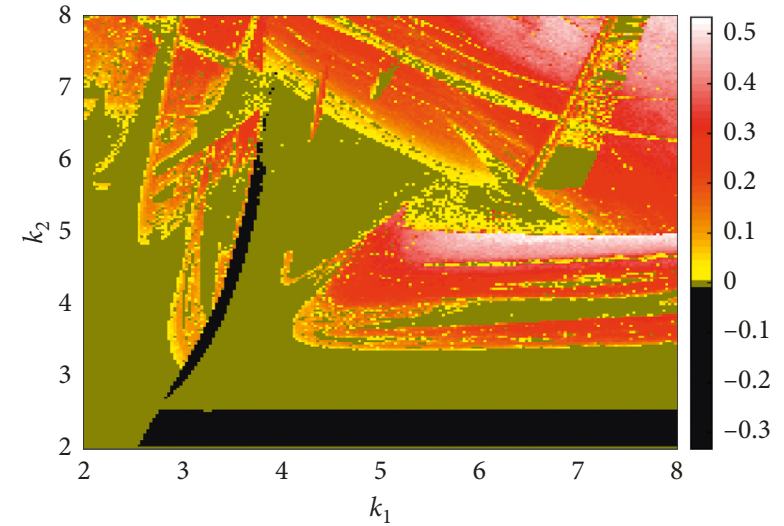

(b)

FIgURe 5: Two-dimensional dynamical maps in the $k_{1}-k_{2}$ parameter plane by evaluating the values of the largest Lyapunov exponent under two sets of initial values. (a) Initial values $\left(10^{-6}, 0,0\right)$. (b) Initial values $\left(10^{-6}, 3,0\right)$. 


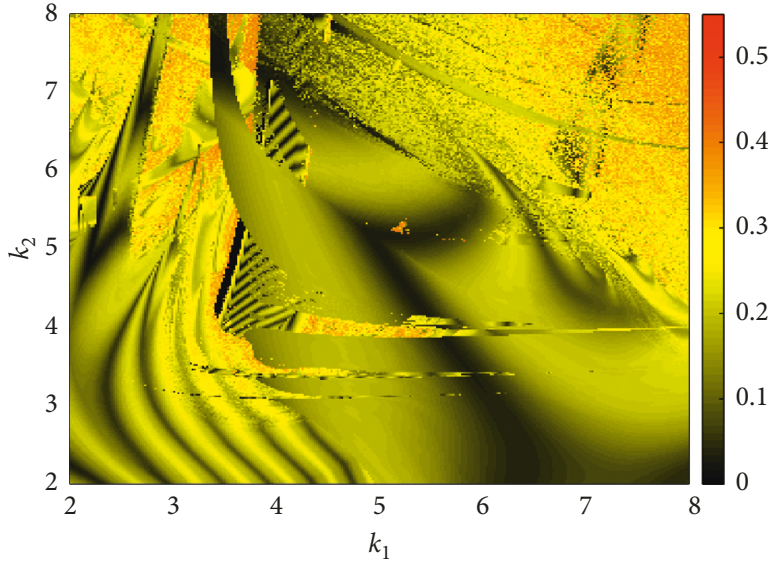

(a)

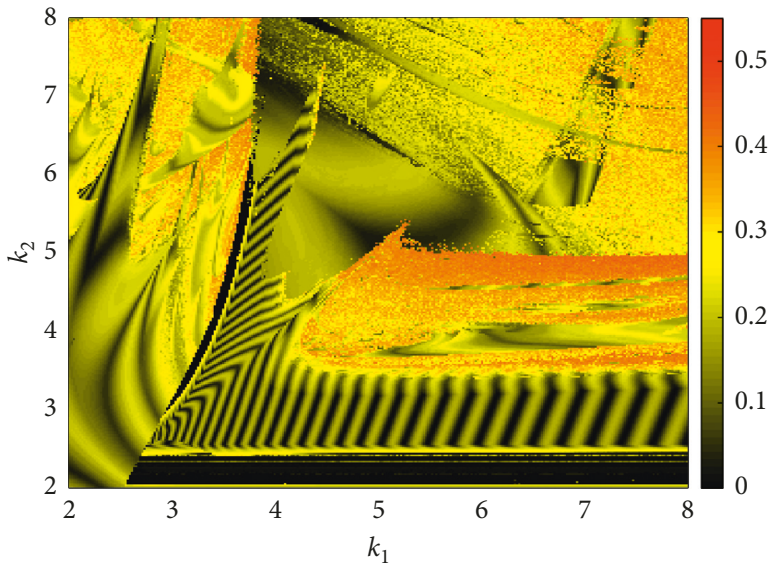

(b)

FIgURE 6: Two-dimensional spectral entropy-based complexity plots for the variable $x$ sequence in the $k_{1}-k_{2}$ parameter plane under two sets of initial values. (a) Initial values $\left(10^{-6}, 0,0\right)$. (b) Initial values $\left(10^{-6}, 3,0\right)$.

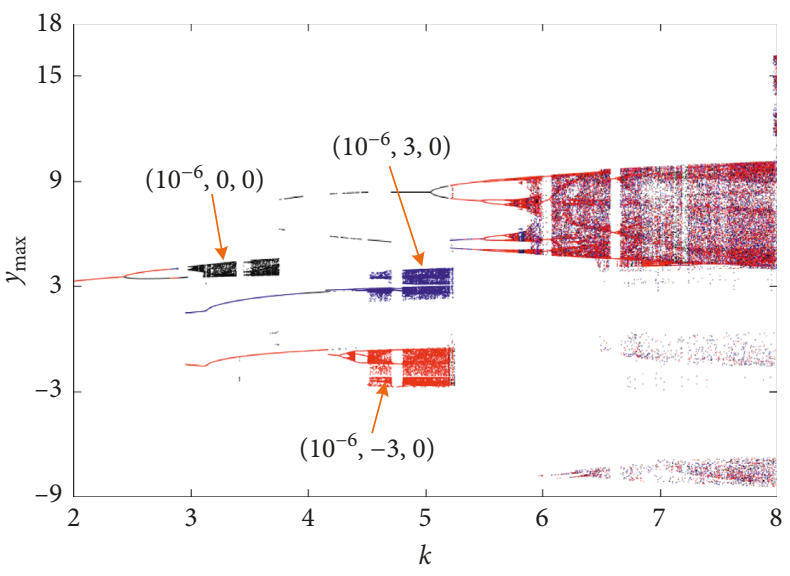

(a)

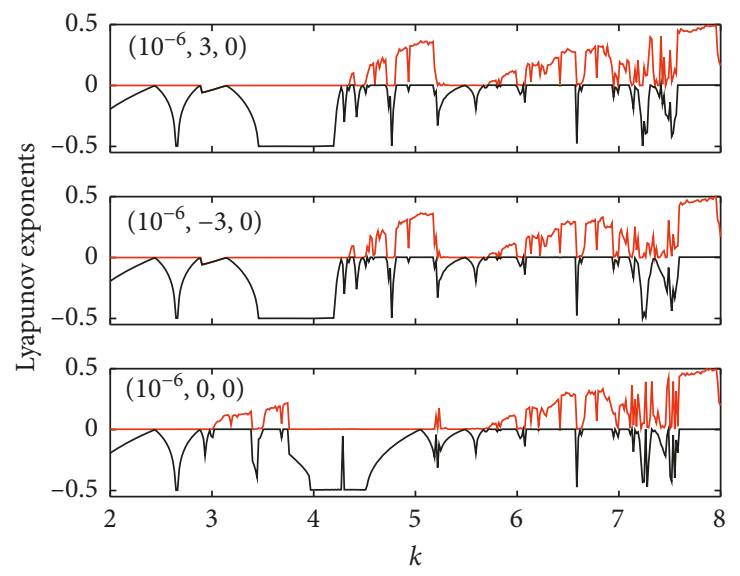

(b)

FIGURE 7: For three sets of initial values $\left(10^{-6}, 0,0\right),\left(10^{-6}, 3,0\right)$, and $\left(10^{-6},-3,0\right)$, one-dimensional bifurcation plots with the variation of the system parameter $k$. (a) Bifurcation diagrams of the maxima $y_{\max }$ of the variable $y$. (b) First two Lyapunov exponents.

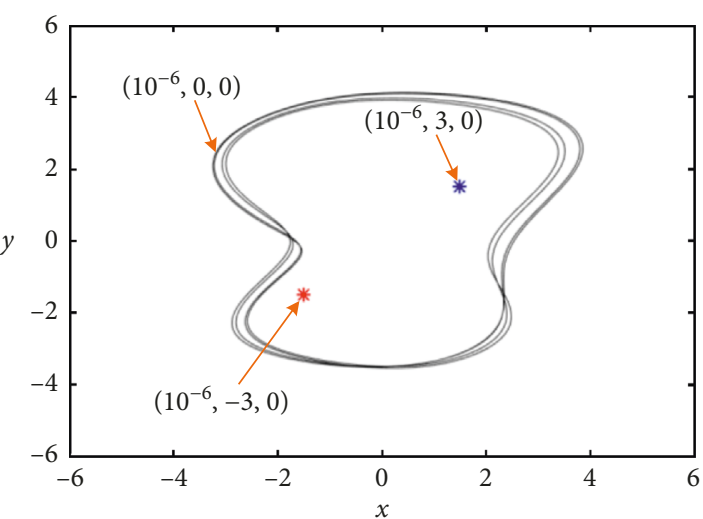

(a)

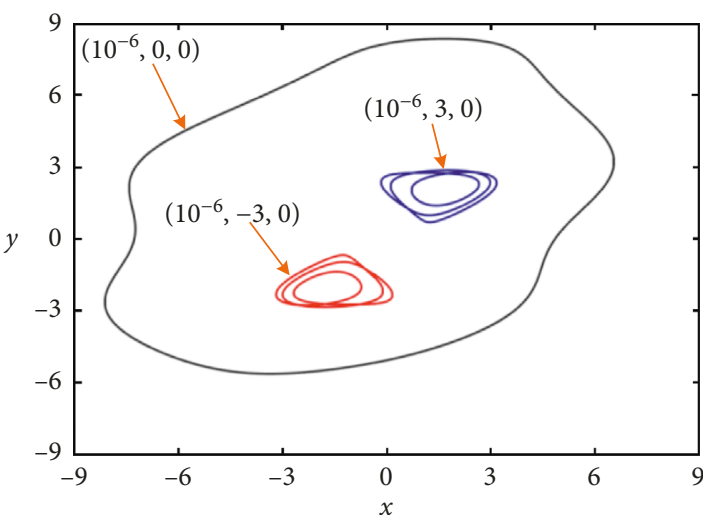

(b)

Figure 8: Continued. 


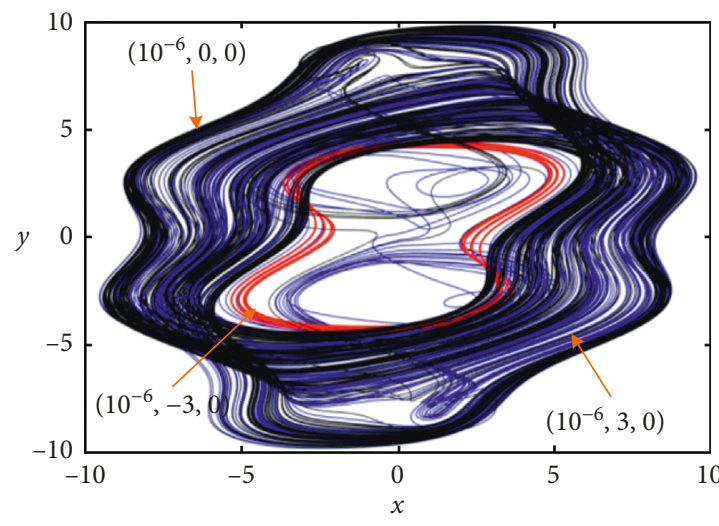

(c)

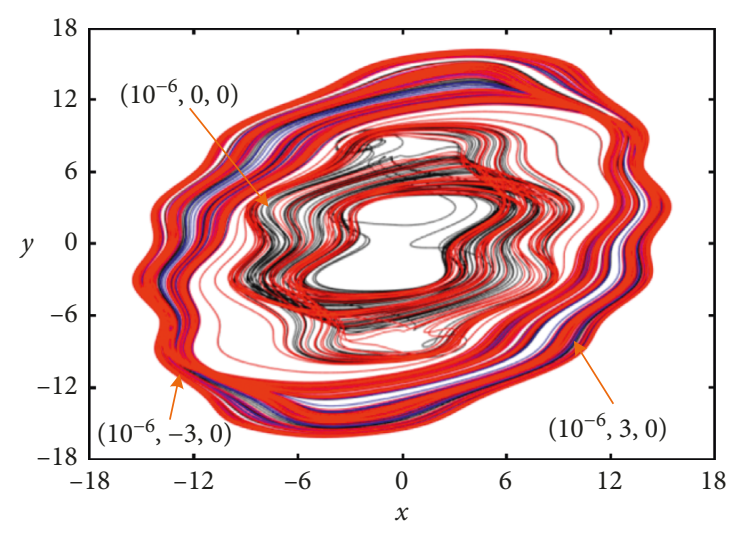

(d)

Figure 8: Phase plots of coexisting attractors in the $x-y$ plane for different values of the parameter $k$. (a) Period- 4 limit cycle coexisted with a pair of symmetric points at $k=3$. (b) Large-size period-1 limit cycle coexisted with a pair of symmetric period-3 limit cycles at $k=4.5$. (c) Chaotic attractor coexisted with period-5 limit cycle at $k=7$. (d) Coexisting chaotic attractors with two topologies at $k=8$.

differences in the parameter region $(6.94,7.58)$. However, when the other two sets of initial values $\left(10^{-6}, 0,0\right)$ and $\left(10^{-6}, 3\right.$, 0 ) are chosen, both the depicted dynamical behaviors in Figure 7 have big differences in the parameter region (2.96, $5.24)$. As the parameter $k$ is increased in this parameter region, the moving orbit for $\left(10^{-6}, 0,0\right)$ goes into chaotic oscillating state at $k=3.01$ via period-doubling bifurcation route and mutates into periodic oscillating state at $k=3.76$ via chaos crisis, whereas the moving orbit for $\left(10^{-6}, 3,0\right)$ turns into periodic oscillating state from stable resting state at $k=3.14$ and enters into chaotic oscillating state at $k=4.34$ via perioddoubling bifurcation route with two relatively larger periodic windows. Of course, in the parameter region $(6.94,7.58)$, some slight differences between the depicted dynamical behaviors under two sets of initial values $\left(10^{-6}, 0,0\right)$ and $\left(10^{-6}, 3,0\right)$ can be seen for the presented sine system as well.

Except for the two examples in Figure 2, other examples to exhibit coexisting multiple attractors' behaviors are given in Figure 8, where four sets of phase plots in the $x-y$ plane are provided together for the initial values $\left(10^{-6}, 0,0\right),\left(10^{-6}, 3\right.$, $0)$, and $\left(10^{-6},-3,0\right)$. When $k=3$, the coexistence of a period4 limit cycle and a pair of symmetric points is exhibited in Figure $8(\mathrm{a})$. When $k=4.5$, the coexistence of a large size period-1 limit cycle and a pair of symmetric period-3 limit cycles is demonstrated in Figure 8(b). When $k=7$, the coexistence of a chaotic attractor and a period-5 limit cycle is illustrated in Figure $8(\mathrm{c})$. However, when $k=8$, the coexistence of two chaotic attractors with different topologies is disclosed in Figure 8(d). Consequently, various types of coexisting attractors' behaviors can be found in the presented sine system.

\section{Validations by the Simulation-Oriented Circuit Model}

By employing PSIM Version 9.0.3 software, the simulationoriented circuit model for implementation of the presented sine system is synthesized and its screen shot is given in Figure 9, in which three operation channels containing three integrators, three inverters and two sine function converters are used to implement three state variables $x, y$, and $z$, respectively.

Based on the simulation-oriented circuit model shown in Figure 9, the state equations for the capacitor voltages $v_{x}, v_{y}$, and $v_{z}$ are described by

$$
\begin{aligned}
& R C \frac{\mathrm{d} v_{x}}{\mathrm{~d} t}=v_{y}+v_{z}-\frac{R}{R_{k 1}} \sin \left(v_{y}\right), \\
& R C \frac{\mathrm{d} v_{y}}{\mathrm{~d} t}=-v_{x}+v_{z}, \\
& R C \frac{\mathrm{d} v_{z}}{\mathrm{~d} t}=-v_{x}-v_{z}-\frac{R}{R_{k 2}} \sin \left(v_{x}\right) .
\end{aligned}
$$

where $C_{1}=C_{2}=C_{3}=C, R_{k 1}=R / k_{1}$, and $R_{k 2}=R / k_{2}$. When $R C=10 \mathrm{k} \Omega \times 10 \mathrm{nF}=100 \mu$ s, i.e., $R=10 \mathrm{k} \Omega$ and $C=10 \mathrm{nF}$, the circuit parameters $R_{k 1}$ and $R_{k 2}$ for PSIM circuit simulations can be conveniently determined.

According to the system parameters $k_{1}$ and $k_{2}$ and the initial values used in Figure 2, the circuit parameters $R_{k 1}$ and $R_{k 2}$ have the same values, i.e., $R_{k 1}=R_{k 2}$. When $R_{k 1}=R_{k 2}=2.78 \mathrm{k} \Omega$ and $2 \mathrm{k} \Omega$, respectively, PSIM screen captures are obtained in Figure 10, where the initial voltages $v_{x}(0)$ and $v_{z}(0)$ of the capacitors $C_{1}$ and $C_{3}$ are always fixed as $1 \mu \mathrm{V}$ and $0 \mathrm{~V}$, respectively, and only the initial voltage $v_{y}(0)$ of the capacitor $C_{2}$ is adjusted as different initial values.

Similarly, based on the system parameters $k_{1}$ and $k_{2}$ and three sets of initial values used in Figure 8, the circuit parameters are selected as $R_{k 1}=R_{k 2}=3.33 \mathrm{k} \Omega, 2.22 \mathrm{k} \Omega, 1.43 \mathrm{k} \Omega$, and $1.25 \mathrm{k} \Omega$, respectively. The corresponding PSIM screen captures are attached in Figure 11, where the initial voltages $v_{x}(0), v_{y}(0)$, and $v_{z}(0)$ of the capacitors $C_{1}, C_{2}$, and $C_{3}$ are assigned as $v_{x}(0)=1 \mu \mathrm{V}, v_{y}(0)=3 \mathrm{~V}$ (or $0 \mathrm{~V}$ and $-3 \mathrm{~V}$ ), and $v_{z}(0)=0 \mathrm{~V}$, respectively.

PSIM circuit simulations in Figure 11(d) are slightly different from MATLAB numerical simulations in Figure $8(d)$, which are mainly caused by the inconsistently 


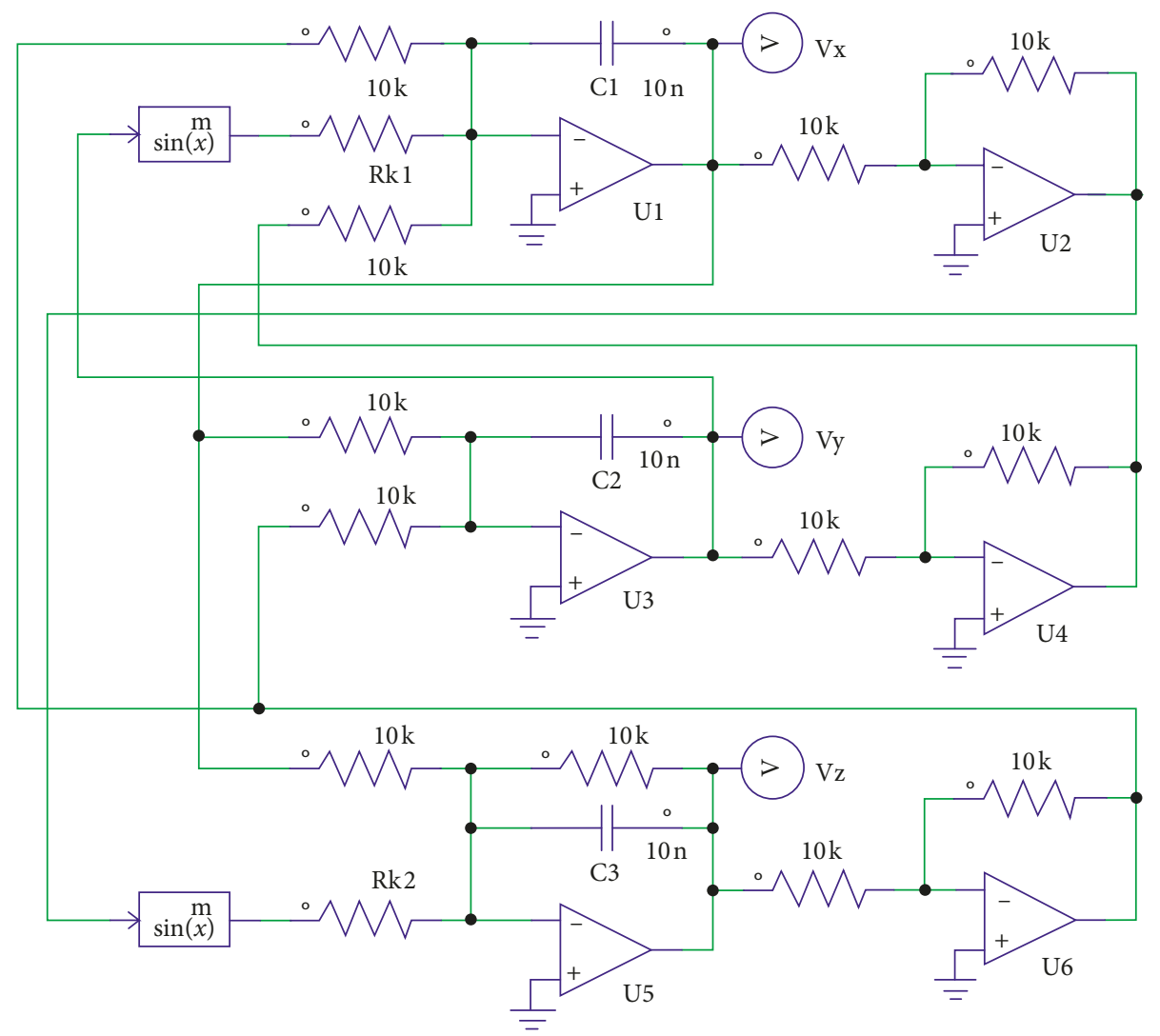

FIgURE 9: Screen shot of PSIM simulation-oriented circuit model for implementation of the presented sine system.

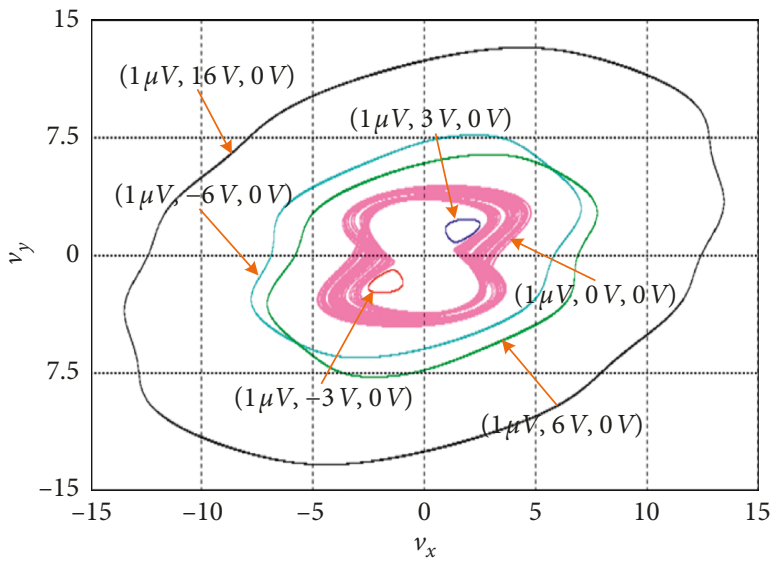

(a)

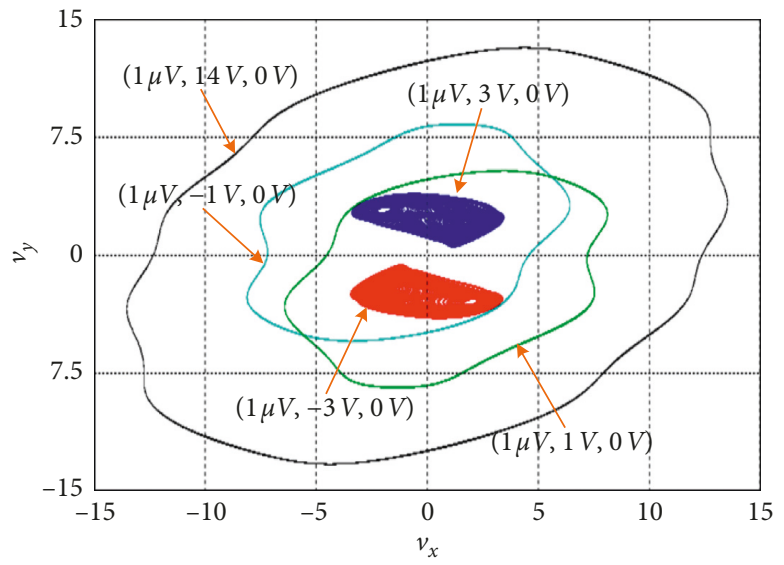

(b)

FIGURE 10: PSIM screen captures of coexisting multiple attractors in the $v_{x}-v_{y}$ plane for different initial values. (a) For $R_{k 1}=R_{k 2}=2.78 \mathrm{k} \Omega$, six types of coexisting multiple attractors. (b) For $R_{k 1}=R_{k 2}=2 \mathrm{k} \Omega$, five types of coexisting multiple attractors.

transient behaviors due to the existence of simulation errors [46]. Ignoring the tiny differences between MATLAB numerical simulations and PSIM circuit simulations, the results in Figures 10 and 11 effectively validate the coexisting attractors' behaviors disclosed in Figures 2 and 8.

Besides, it should be mentioned that the sine function terms are the two key units for realizing the proposed 3-D sine chaotic system. In the analog circuit experiments [47], the sine function terms can be physically implemented using two AD639AD trigonometric function converters. But the system initials, corresponding to the initial capacitor voltages, are hardly set in the experimental measurements. In contrast, in the digital circuit experiments [48], the sine function terms can be directly achieved by calling IP cores in CORDIC library of FPGA and the system initials can be readily preset. Therefore, a feasible way to realize the proposed 3-D sine chaotic system could be implemented on the FPGA, which is addressed in our future paper. 


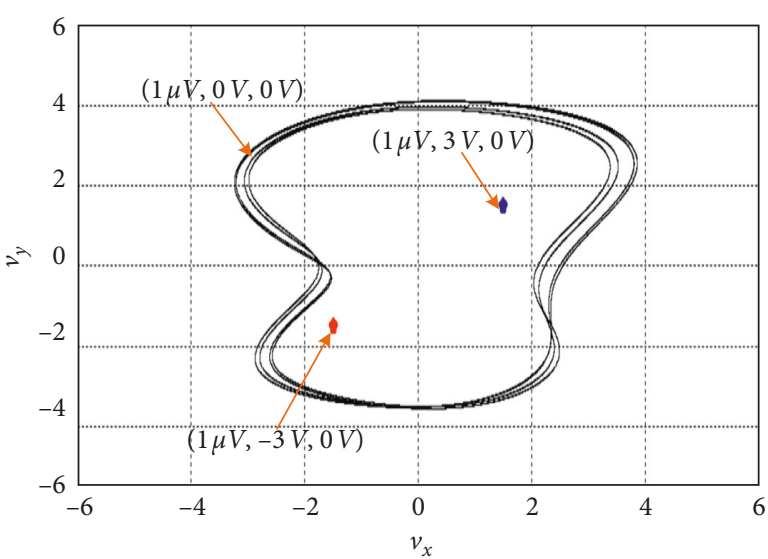

(a)

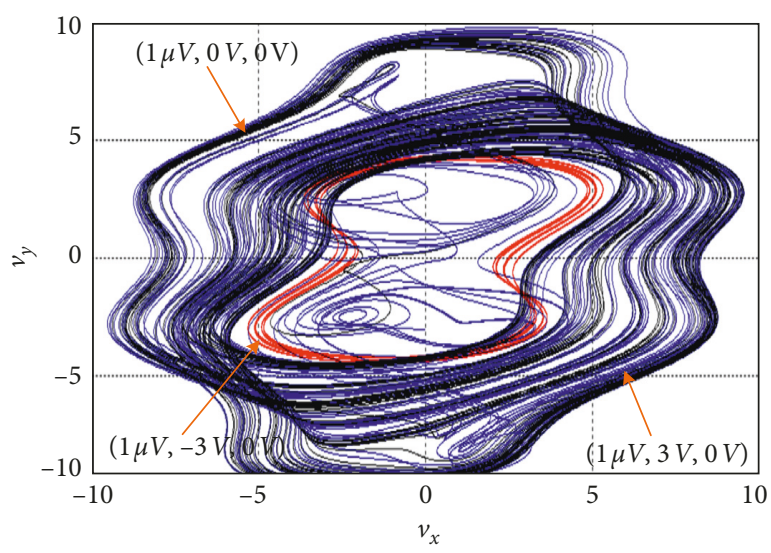

(c)

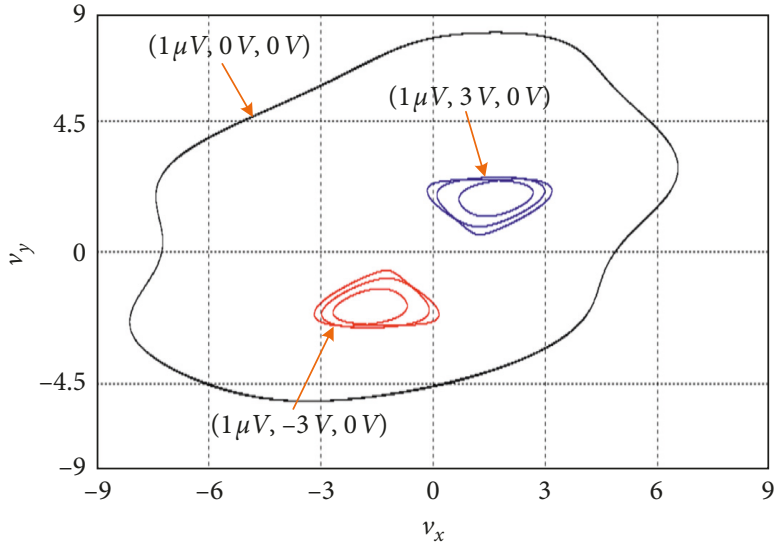

(b)

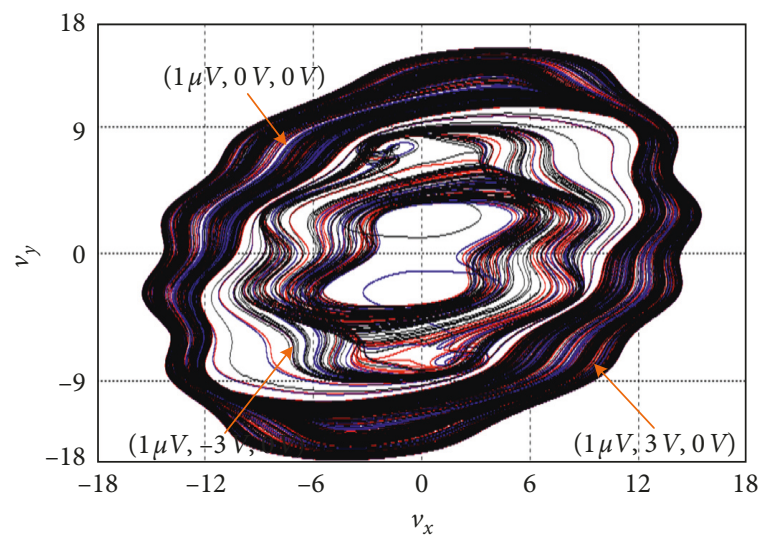

(d)

FIgURE 11: PSIM screen captures of coexisting attractors in the $v_{x}-v_{x}$ plane for different values of $R_{k 1}$ and $R_{k 2}$. (a) Period-4 limit cycle coexisted with a pair of symmetric points at $R_{k 1}=R_{k 2}=3.33 \mathrm{k} \Omega$. (b) Large-size period-1 limit cycle coexisted with a pair of symmetric period3 limit cycles at $R_{k 1}=R_{k 2}=2.22 \mathrm{k} \Omega$. (c) Chaotic attractor coexisted with period-5 limit cycle at $R_{k 1}=R_{k 2}=1.43 \mathrm{k} \Omega$. (d) Coexisting chaotic attractors with two topologies at $R_{k 1}=R_{k 2}=1.25 \mathrm{k} \Omega$.

\section{Conclusion}

The autonomous chaotic systems can generate the conventional self-excited attractors as their oscillations are excited from the unstable determined equilibrium points. The mechanism for constructing chaotic systems with coexisting multiple attractors is based on the fact that the system equilibrium points can be reinstalled by newly introduced sine nonlinearities, leading to the great variations of their number, characteristics, and distributions [49]. Therefore, by introducing two sine nonlinearities into a simple 3-D linear dynamical system, this paper presented a novel and simple 3-D sine chaotic system with the reinstalled five index- 2 saddle foci and four index- 1 saddle foci, from which the abundant coexisting multiple attractors' behaviors were thereby revealed by numerical simulations, such as phase plots, attraction basins, two-dimensional bifurcation and complexity plots, and one-dimensional bifurcation plots, and finally validated by PSIM circuit simulations. The algebraic simplicity of system structure and topological complexity of chaotic attractor are a long-term goal for seeking a new chaotic system with coexisting behaviors, which could acquire wide interest for its chaos-based engineering applications $[42,50]$.

\section{Data Availability}

The data used to support the findings of this study are available from the corresponding author upon request.

\section{Conflicts of Interest}

The authors declare that they have no conflicts of interest.

\section{Acknowledgments}

This research was supported by the grants from the National Natural Science Foundations of China under Grant nos. 
51607013, 61601062, and 61801054 and Natural Science Foundation of Jiangsu Province, China, under Grant no. BK20191451.

\section{References}

[1] G. H. Kom, J. Kengne, J. R. Mboupda Pone, G. Kenne, and A. B. Tiedeu, "Asymmetric double strange attractors in a simple autonomous jerk circuit," Complexity, vol. 2018, Article ID 4658785, 16 pages, 2018.

[2] L. Zhou, C. H. Wang, X. Zhang, and W. Yao, "Various attractors, coexisting attractors and antimonotonicity in a simple fourth-order memristive Twin-T oscillator," International Journal of Bifurcation and Chaos, vol. 28, no. 4, Article ID 1850050, 2018.

[3] M. Chen, Q. Xu, Y. Lin, and B. Bao, "Multistability induced by two symmetric stable node-foci in modified canonical Chua's circuit," Nonlinear Dynamics, vol. 87, no. 2, pp. 789-802, 2017.

[4] A. T. Azar, N. M. Adele, T. Alain, R. Kengne, and F. H. Bertrand, "Multistability analysis and function projective synchronization in relay coupled oscillators," Complexity, vol. 2018, Article ID 3286070, 12 pages, 2018.

[5] N. Stankevich and E. Volkov, "Multistability in a three-dimensional oscillator: tori, resonant cycles and chaos," Nonlinear Dynamics, vol. 94, no. 4, pp. 2455-2467, 2018.

[6] B. C. Bao, A. H. Hu, H. Bao, Q. Xu, M. Chen, and H. G. Wu, "Three-dimensional memristive Hindmarsh-Rose neuron model with hidden coexisting asymmetric behaviors," Complexity, vol. 2018, Article ID 3872573, 11 pages, 2018.

[7] H. Bao, W. Liu, and A. Hu, "Coexisting multiple firing patterns in two adjacent neurons coupled by memristive electromagnetic induction," Nonlinear Dynamics, vol. 95, no. 1, pp. 43-56, 2019.

[8] Z. T. Njitacke and J. Kengne, "Complex dynamics of a 4D Hopfield neural networks (HNNs) with a nonlinear synaptic weight: coexistence of multiple attractors and remerging Feigenbaum trees," AEU-International Journal of Electronics and Communications, vol. 93, pp. 242-252, 2018.

[9] B. C. Bao, H. Qian, Q. Xu, M. Chen, J. Wang, and Y. J. Yu, "Coexisting behaviors of asymmetric attractors in hyperbolictype memristor based Hopfield neural network," Frontiers in Computational Neuroscience, vol. 11, no. 81, pp. 1-14, 2017.

[10] K. Rajagopal, J. M. Munoz-Pacheco, V.-T. Pham, D. V. Hoang, F. E. Alsaadi, and F. E. Alsaadi, "A Hopfield neural network with multiple attractors and its FPGA design," The European Physical Journal Special Topics, vol. 227, no. 7-9, pp. 811-820, 2018.

[11] C. Chen, J. Chen, H. Bao, M. Chen, and B. Bao, "Coexisting multi-stable patterns in memristor synapse-coupled Hopfield neural network with two neurons," Nonlinear Dynamics, vol. 95, no. 4, pp. 3385-3399, 2019.

[12] Y. Zhang and G. Luo, "Multistability of a three-degree-offreedom vibro-impact system," Communications in Nonlinear Science and Numerical Simulation, vol. 57, pp. 331-341, 2018.

[13] J. Hizanidis, N. Lazarides, and G. P. Tsironis, "Flux biascontrolled chaos and extreme multistability in SQUID oscillators," Chaos: An Interdisciplinary Journal of Nonlinear Science, vol. 28, no. 6, Article ID 063117, 2018.

[14] Q. Lai, P. D. K. Kuate, F. Liu, and H. H. C. Iu, "An extremely simple chaotic system with infinitely many coexisting attractors," IEEE Transactions on Circuits and Systems II: Express Briefs, 2019.
[15] M. Chen, Y. Feng, H. Bao et al., "State variable mapping method for studying initial-dependent dynamics in memristive hyper-jerk system with line equilibrium," Chaos, Solitons \& Fractals, vol. 115, pp. 313-324, 2018.

[16] Z. T. Njitacke, J. Kengne, R. W. Tapche, and F. B. Pelap, "Uncertain destination dynamics of a novel memristive 4D autonomous system," Chaos, Solitons \& Fractals, vol. 107, pp. 177-185, 2018.

[17] H. Bao, N. Wang, B. Bao, M. Chen, P. Jin, and G. Wang, "Initial condition-dependent dynamics and transient period in memristor-based hypogenetic jerk system with four line equilibria," Communications in Nonlinear Science and $\mathrm{Nu}$ merical Simulation, vol. 57, pp. 264-275, 2018.

[18] A. N. Pisarchik and U. Feudel, "Control of multistability," Physics Reports, vol. 540, no. 4, pp. 167-218, 2014.

[19] P. R. Sharma, M. D. Shrimali, A. Prasad, N. V. Kuznetsov, and G. A. Leonov, "Control of multistability in hidden attractors," The European Physical Journal Special Topics, vol. 224, no. 8, pp. 1485-1491, 2015.

[20] M. Chen, M. Sun, B. Bao, H. Wu, Q. Xu, and J. Wang, "Controlling extreme multistability of memristor emulatorbased dynamical circuit in flux-charge domain," Nonlinear Dynamics, vol. 91, no. 2, pp. 1395-1412, 2018.

[21] F. Hegedüs, W. Lauterborn, U. Parlitz, and R. Mettin, "Nonfeedback technique to directly control multistability in nonlinear oscillators by dual-frequency driving," Nonlinear Dynamics, vol. 94, no. 1, pp. 273-293, 2018.

[22] K. Yadav, A. Prasad, and M. D. Shrimali, "Control of coexisting attractors via temporal feedback," Physics Letters A, vol. 382, no. 32, pp. 2127-2132, 2018.

[23] M. Chen, M. X. Sun, H. Bao, Y. H. Hu, and B. C. Bao, "Fluxcharge analysis of two-memristor-based Chua's circuit: dimensionality decreasing model for detecting extreme multistability," IEEE Transactions on Industrial Electronics, vol. 67, no. 3, pp. 2197-2206, 2019.

[24] Z. Wang, A. Akgul, V.-T. Pham, and S. Jafari, "Chaos-based application of a novel no-equilibrium chaotic system with coexisting attractors," Nonlinear Dynamics, vol. 89, no. 3, pp. 1877-1887, 2017.

[25] Q. Lai, B. Norouzi, and F. Liu, "Dynamic analysis, circuit realization, control design and image encryption application of an extended Lü system with coexisting attractors," Chaos, Solitons \& Fractals, vol. 114, pp. 230-245, 2018.

[26] G. Peng and F. Min, "Multistability analysis, circuit implementations and application in image encryption of a novel memristive chaotic circuit," Nonlinear Dynamics, vol. 90, no. 3, pp. 1607-1625, 2017.

[27] C. Li, F. H. Min, Q. S. Jin, and H. Y. Ma, "Extreme multistability analysis of memristor-based chaotic system and its application in image decryption," AIP Advances, vol. 7, no. 12, Article ID 125204, 2017.

[28] F. Yuan, G. Y. Wang, and X. W. Wang, "Chaotic oscillator containing memcapacitor and meminductor and its dimensionality reduction analysis," Chaos: An Interdisciplinary Journal of Nonlinear Science, vol. 27, no. 3, Article ID 033103, 2017.

[29] M. Chen, Y. Feng, H. Bao, B. C. Bao, H. G. Wu, and Q. Xu, "Hybrid state variable incremental integral for reconstructing extreme multistability in memristive jerk system with cubic nonlinearity," Complexity, vol. 2019, Article ID 8549472, 16 pages, 2019.

[30] H. Bao, T. Jiang, K. B. Chu, M. Chen, Q. Xu, and B. C. Bao, "Memristor-based canonical Chua's circuit: extreme multistability in voltage-current domain and its controllability in 
flux-charge domain," Complexity, vol. 2018, Article ID 5935637, 13 pages, 2018.

[31] M. Chen, B. C. Bao, T. Jiang et al., "Flux-Charge analysis of initial state-dependent dynamical behaviors of a memristor emulator-based chua's circuit," International Journal of Bifurcation and Chaos, vol. 28, no. 10, Article ID 1850120, 2018.

[32] H. Bao, W. Liu, and M. Chen, "Hidden extreme multistability and dimensionality reduction analysis for an improved nonautonomous memristive FitzHugh-Nagumo circuit," Nonlinear Dynamics, vol. 96, no. 3, pp. 1879-1894, 2019.

[33] Q. Xu, Y. Lin, B. Bao, and M. Chen, "Multiple attractors in a non-ideal active voltage-controlled memristor based Chua's circuit," Chaos, Solitons \& Fractals, vol. 83, pp. 186-200, 2016.

[34] B. Bao, T. Jiang, G. Wang, P. Jin, H. Bao, and M. Chen, "Twomemristor-based Chua's hyperchaotic circuit with plane equilibrium and its extreme multistability," Nonlinear Dynamics, vol. 89, no. 2, pp. 1157-1171, 2017.

[35] L. Wang, S. Zhang, Y.-C. Zeng, and Z.-J. Li, "Generating hidden extreme multistability in memristive chaotic oscillator via micro-perturbation," Electronics Letters, vol. 54, no. 13, pp. 808-810, 2018.

[36] J. Kengne, Z. T. Njitacke, and H. B. Fotsin, "Dynamical analysis of a simple autonomous jerk system with multiple attractors," Nonlinear Dynamics, vol. 83, no. 1-2, pp. 751-765, 2016.

[37] C. Li and J. C. Sprott, "An infinite 3-D quasiperiodic lattice of chaotic attractors," Physics Letters A, vol. 382, no. 8, pp. 581-587, 2018.

[38] J. Sun, X. Zhao, J. Fang, and Y. Wang, "Autonomous memristor chaotic systems of infinite chaotic attractors and circuitry realization," Nonlinear Dynamics, vol. 94, no. 4, pp. 2879-2887, 2018.

[39] C. Li, Y. Xu, G. Chen, Y. Liu, and J. Zheng, "Conditional symmetry: bond for attractor growing," Nonlinear Dynamics, vol. 95, no. 2, pp. 1245-1256, 2019.

[40] Q. Lai, C. Chen, X.-W. Zhao, J. Kengne, and C. Volos, "Constructing chaotic system with multiple coexisting attractors," IEEE Access, vol. 7, pp. 24051-24056, 2019.

[41] C. Li, W. Joo-Chen Thio, J. C. Sprott, H. H.-C. Iu, and Y. Xu, "Constructing infinitely many attractors in a programmable chaotic circuit," IEEE Access, vol. 6, pp. 29003-29012, 2018.

[42] G. Alvarez and S. Li, "Some basic cryptographic requirements for chaos-based cryptosystems," International Journal of Bifurcation and Chaos, vol. 16, no. 8, pp. 2129-2151, 2006.

[43] T. F. Fonzin, K. Srinivasan, J. Kengne, and F. B. Pelap, "Coexisting bifurcations in a memristive hyperchaotic oscillator," AEÜ-International Journal of Electronics and Communications, vol. 90, pp. 110-122, 2018.

[44] C. C. Strelioff and A. W. Hübler, "Medium-term prediction of chaos," Physical Review Letters, vol. 96, no. 4, Article ID 044101, 2006.

[45] H. Bao, M. Chen, H. Wu, and B. Bao, "Memristor initialboosted coexisting plane bifurcations and its extreme multistability reconstitution in two-memristor-based dynamical system," Science China Technological Sciences, 2019.

[46] N. V. Kuznetsov, G. A. Leonov, M. V. Yuldashev, and R. V. Yuldashev, "Hidden attractors in dynamical models of phase-locked loop circuits: limitations of simulation in MATLAB and SPICE," Communications in Nonlinear Science and Numerical Simulation, vol. 51, pp. 39-49, 2017.

[47] Q. Lai, A. Akgul, C. Li, G. Xu, and Ü. Çavuşoğlu, "A new chaotic system with multiple attractors: dynamic analysis, circuit realization and S-Box design," Entropy, vol. 20, no. 1, p. 12, 2018.
[48] B. C. Bao, Q. F. Yang, L. Zhu et al., "Chaotic bursting dynamics and coexisting multistable firing patterns in $3 \mathrm{D}$ autonomous Morris-Lecar model and microcontroller-based validations," International Journal of Bifurcation and Chaos, vol. 29, no. 10, Article ID 1950134, 2019.

[49] V. T. Pham, C. Volos, T. Kapitaniak, S. Jafari, and X. Wang, "Dynamics and circuit of a chaotic system with a curve of equilibrium points," International Journal of Electronics, vol. 105, no. 3, pp. 385-397, 2018.

[50] Z. Y. Hua, Y. C. Zhou, and B. C. Bao, "Two-dimensional sine chaotification system with hardware implementation," IEEE Transactions on Industrial Informatics, 2019. 


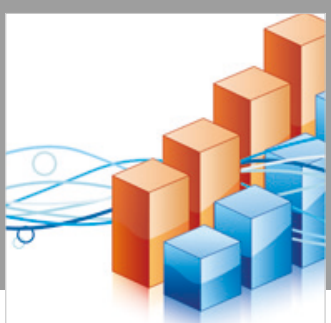

Advances in

Operations Research

\section{-n-m}
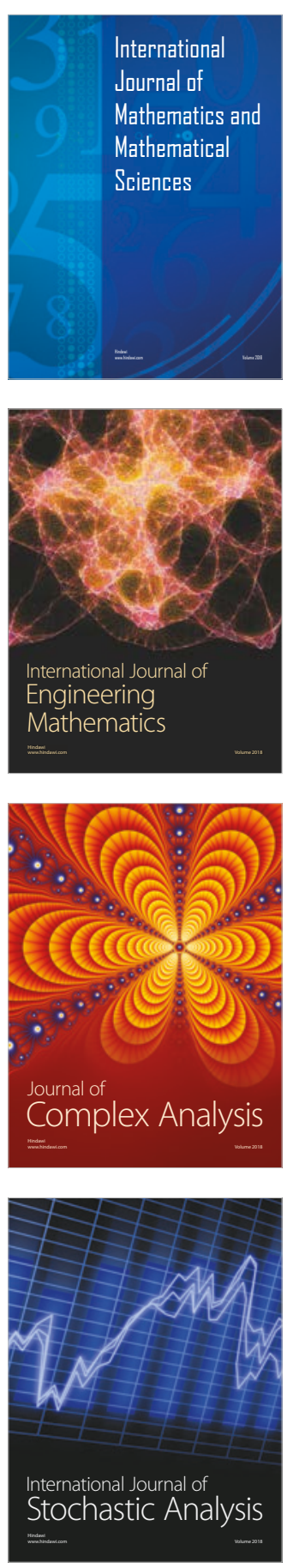
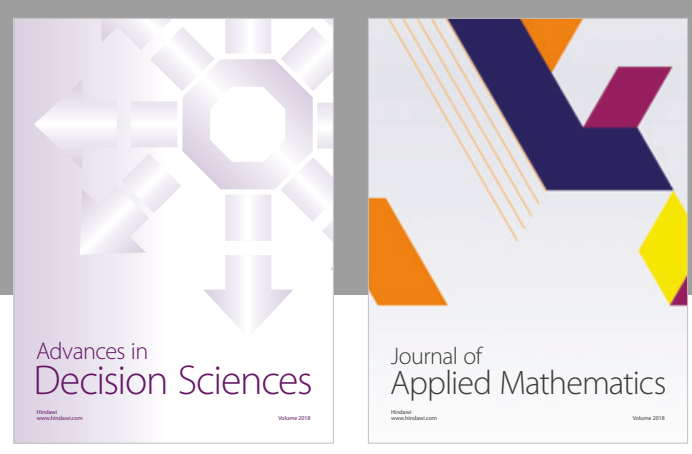

Journal of

Applied Mathematics
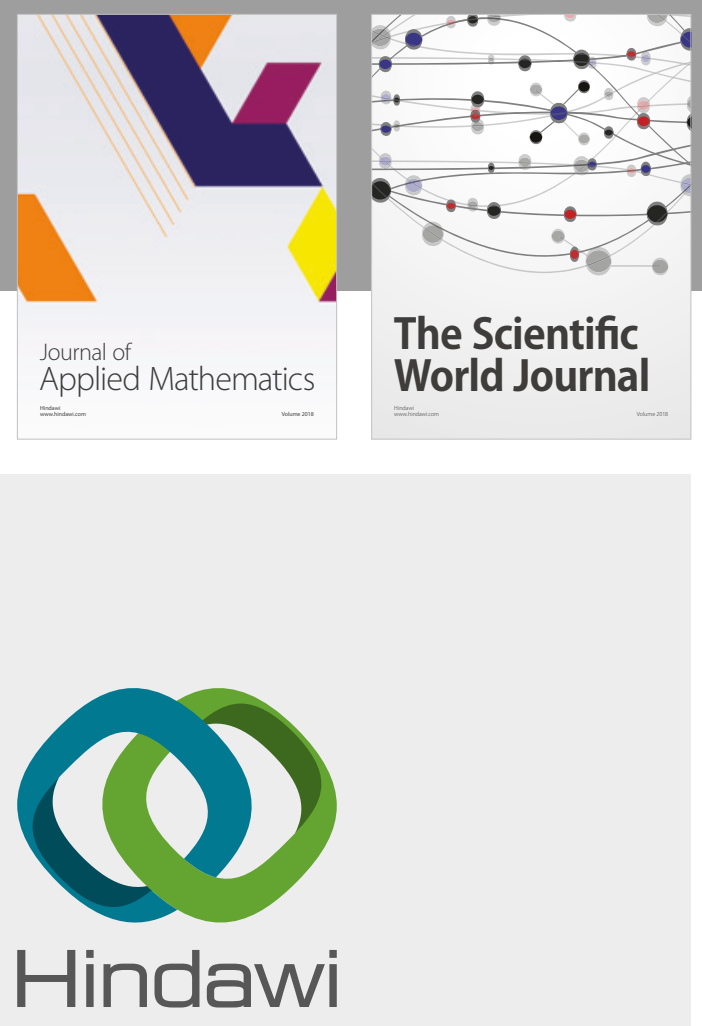

Submit your manuscripts at

www.hindawi.com

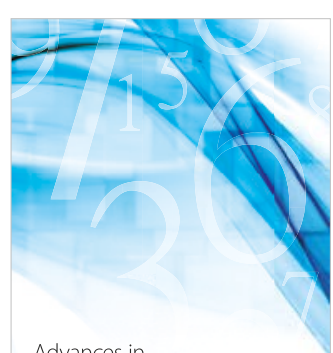

Advances in
Numerical Analysis
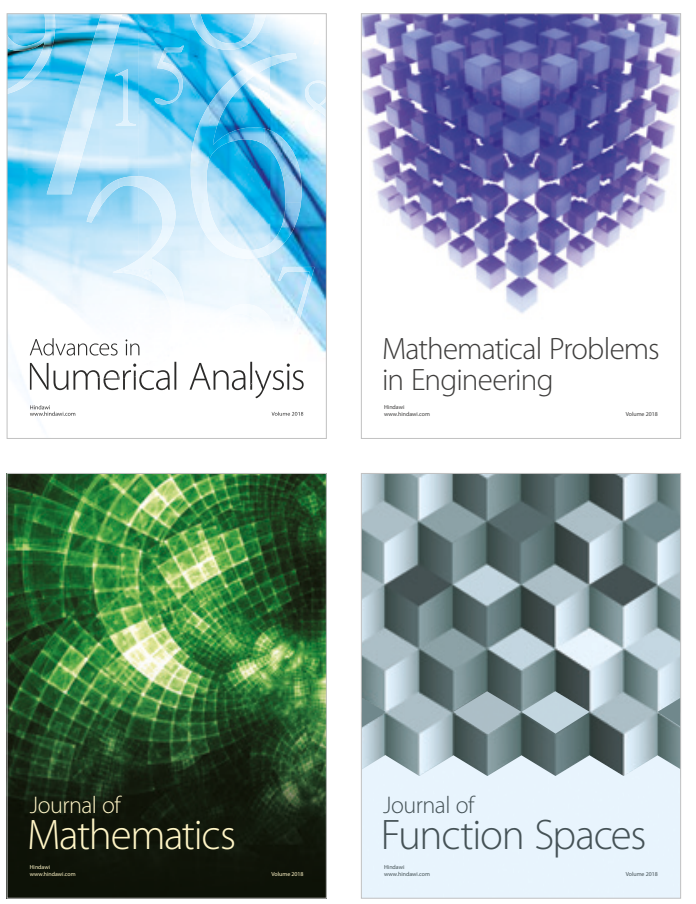

Mathematical Problems in Engineering

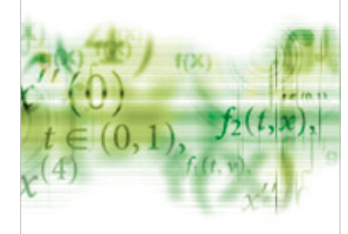

International Journal of

Differential Equations

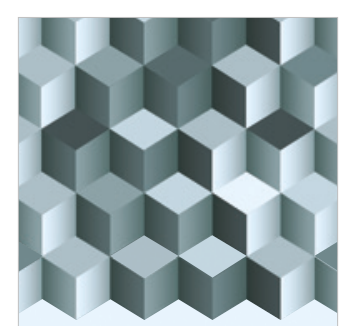

Journal of

Function Spaces
The Scientific

World Journal

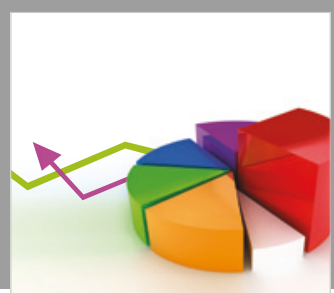

Journal of

Probability and Statistics
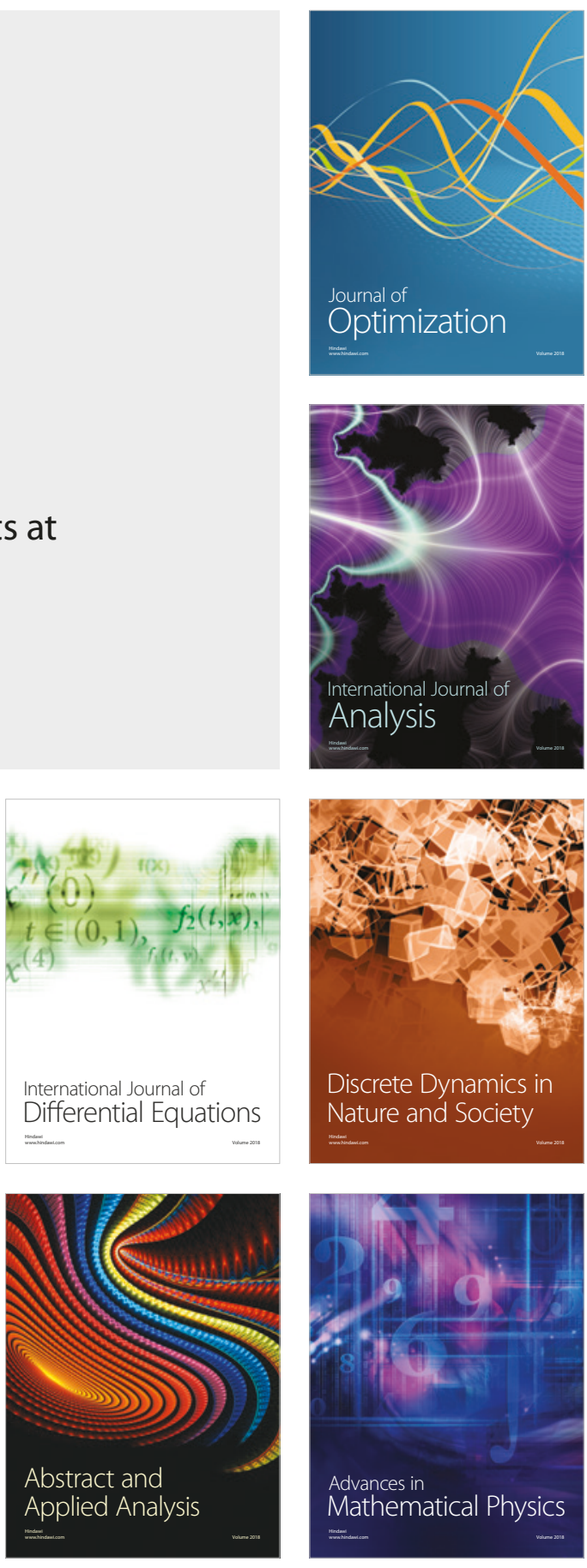